CAJA COSTARRICENSE DE

SEGURO SOCIAL

HOSPITAL SAN JUAN DE

DIOS

SERVICIO DE FARMACIA

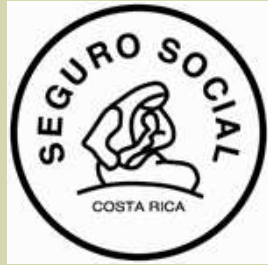

\section{"Se ha asumido que}

una propiedad

común a todos los

excipientes emplea-

dos en una formula-

ción de medicamen-

tos es la inercia"

CONTENIDO:

Concepto de exci- ।

pientes

Reacciones adversas 2 asociadas a determi-

nados excipientes

Ejemplos y lista de 3 excipientes de reporte obligatorio

Alertas de Farmaco- 5 vigilancia enero $y$

febrero 2013

\title{
Reacciones adversas a
}

excipientes

VOL U M E N 3, N Ú MERO I

E N E R O Y F E B R E R O

\section{Introducción: Concepto de excipientes}

Se entiende por excipiente a la sustancia $\circ$ mezcla de sustancias inactivas por sí mismas, pero que están presentes en las especialidades farmacéuticas con el objetivo de estabilizar, colorear, saborizar o modificar las propiedades biofarmacéuticas del medicamento.

Los excipientes constituyen una fracción importante de las especialidades farmacéuticas, hasta el punto de llegar a representar la mayor parte de la masa o volumen de las mismas.

Existe además un considerable número de excipientes debido a la diversidad de las características de los principios activos a los que deben adaptarse.

A pesar de la cantidad y variedad de excipientes empleados en la formulación de medicamentos, siempre se ha asumido que una propiedad común a todos ellos es la inercia, tanto con el principio activo, como con el material de acondicionamiento y con el organismo.

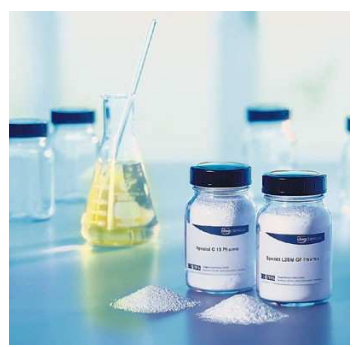

Sin embargo, la neutralidad absoluta respecto al organismo no existe, lo cuál queda evidenciado al consultar la literatura disponi- ble sobre reacciones adversas producidas por excipientes.

Después del año 2003, algunos países europeos lograron modificar su "Ley de Utilización del Medicamento" ordenando a la industria a declarar en el etiquetado del medicamento aquellos excipientes de reporte obligatorio (determinados por la Agencia Europea del Medicamento)

Este boletín está dedicado a la farmacovigilancia de los excipientes, con el objetivo de informar cuáles son los excipientes más asociados a aparición de reacciones adversas y presentar algunos casos documentados en la literatura.

\section{Reacciones adversas asociadas a determinados excipientes}

Como se mencionó en la introducción, la mayoría de los medicamentos son combinaciones de varios productos. Además de los principios activos, los medicamentos contienen numerosos productos aparentemente "inertes" llamados excipientes o aditivos. Según su papel en el medicamento acabado, los excipientes se clasifican en diluentes, edulcorantes, colorantes, preservantes, disgregantes, entre otros.

A continuación se realiza una revisión de las reacciones adversas asociadas con los diferentes tipos de excipientes.

\section{Diluentes}

Se emplean en formulación con el objetivo de proporcionar volumen o masa en preparaciones orales sólidas como tabletas y cápsulas y en preparaciones líquidas como jarabes para aumentar la solubilidad y /o estabilidad del principio activo.
Ejemplos de diluentes: la lactosa, el almidón, el alcohol etílico, el glicerol y el propilenglicol.

La lactosa: Se han descrito casos de intolerancia a este componente en niños y adolescentes, originando cuadros de diarreas complicadas con infección intestinal, deshidratación y acidosis. Aunque la severidad de la intolerancia es variable, en algunas personas pueden manifestarse estos síntomas tan solo después 




"El aspartame es

una fuente de

fenilalanina, por lo

que su ingesta

debe estar

restringida en los

fenilcetonúricos"

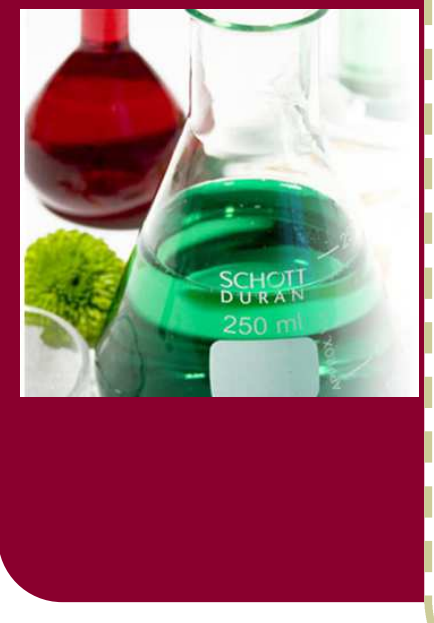

lleguen a causar efectos adversos significativos.

Algunos edulcorantes utilizados son: sacarina, aspartame, sacarosa, entre otros.

Sacarina: a pesar de no ser un excipiente de reporte obligatorio, está presente en cantidades importantes en las formas masticables. Además se sabe que la sacarina es un derivado sulfonado que presenta reactividad cruzada con las sulfonamidas, lo cual quiere decir que los individuos con alergia a las sulfamidas deberían evitar las formulaciones con sacarina como excipiente.

Aspartame: se ha asociado con dolores de cabeza, crisis epilépticas, alteraciones del humor y de la conducta o incluso con el desarrollo de tumores cerebrales, sin embargo, no se han conducido estudios formales para establecer causalidad.

Además, el aspartame es una fuente de fenilalanina, por lo que su ingesta debe estar restringida en en los fenilcetonúricos.

\section{Aspartic}

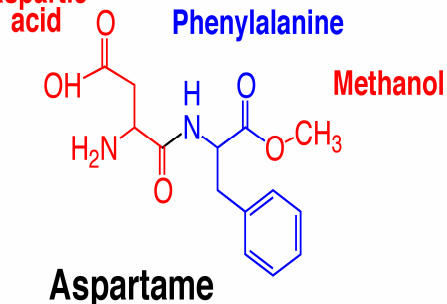

Sacarosa: es el edulcorante más empleado y se considera segura a excepción de algunas comunicaciones relativas al desarrollo de caries dentales, también su administración en pacientes diabéticos puede interferir con el control de la enfermedad. La sacarosa es un excipiente de reporte obligatorio y se exige cuando la dosis máxima diaria constituya un aporte mayor de los 5 gramos. Se acepta que un diabético puede consumir de 10 a 14 gramos de sacarosa sin ser causa de descompensación del equilibrio glucémico.

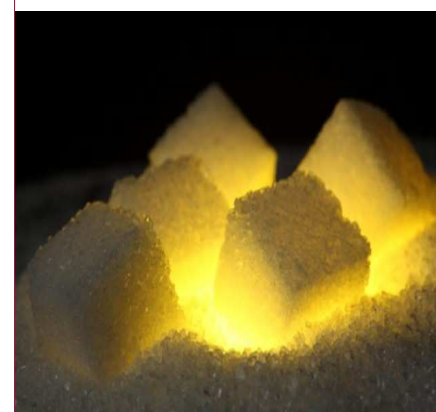

Otros edulcorantes (Manitol, Sorbitol o Xilitol): se atribuyen reacciones adversas de los polialcoholes como diarrea osmótica cuando se consumen en cantidades importantes $\circ$ en pacientes con funcionalidad intestinal reducida.

\section{Colorantes}

Proporcionan a las formulaciones una apariencia característica que contribuye a su identificación.

Los colorantes azoicos se han asociado a reacciones de tipo alérgico e inclusive asma bronquial. La tartrazina es el más común y se considera aún más peligroso en personas con intolerancia a la aspirina. Otros colorantes azolicos son el amaranto, la eritrosina, el rojo Ponceau o el amarilloanaranjado $\mathbf{S}$.

Estos colorantes no sólo se encuentran en productos Farmacéuticos, sino también en derivados lácteos, jugos, caramelos, alimentos, cosméticos y artículos de perfumería. 


\section{Continuación... Reacciones adversas asociadas a determinados excipientes}



cipios activos en la formulación.

Algunos preservantes son: el timerosal, el alcohol bencílico, el cloruro de benzalconio.

Timerosal: es un derivado del mercurio, preservante de algunas vacunas, inmunoglobulinas, preparados oftalmológicos y productos de aplicación tópica. Su administración en pequeñas dosis puede producir sensibilización, dando lugar a reacciones como urticaria, exantema o dermatitis. A concentraciones elevadas presenta toxicidad neurológica y renal. En los últimos años la FDA ha prohibido su presencia en vacunas por suponer una carga de mercurio para los niños, principalmente aquellos menores de 6 meses.

Alcohol bencílico: se ha utilizado ampliamente en la industria Farma céutica por poseer propiedades bacteriostáticas. Se ha descrito reacciones fatales tras su administración parenteral.

Cloruro de benzalconio: preservante muy utilizado en soluciones oftálmicas, sin embargo, su uso continuado produce irritación e hipersensibilidad. También puede estar presente en ciertos nebulizadores presentando riesgo de broncoconstricción en asmáticos.

Sulfitos y metabisulfitos: se utiliza cuando el principio activo de la formulación es inestable en presencia de oxígeno debido a que son antioxidantes. Con ellos se han reportado reacciones de tipo alérgico. La prevalencia de sensibilidad a los sulfitos en la población general no parece ser elevada, pero aumenta considerablemente entre los

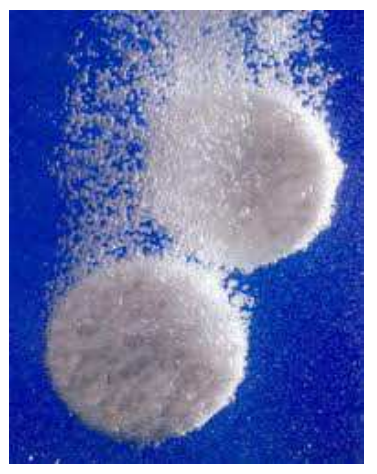

individuos asmáticos. Un ejemplo de una etiqueta de un medicamento que contiene sulfitos es: "Por contener sulfitos como excipiente, su ingesta puede producir manifestaciones alérgicas como disnea, urticaria, angioedema y exacerbación de los broncoespasmos, especialmente en pacientes con asma aguda."

\section{Desinterantes}

En comprimidos efervescentes se requiere para su formulación. Actúan formando un ácido orgánico, que al actuar sobre un carbonato libere el anhídrido carbónico necesario para conseguir la adecuada desintegración de la forma farmacéutica.

Cuando los carbonatos utilizados sean el bicarbonato de sodio o potasio, existirá un aporte extra de estas sales que puede ser poco conveniente en individuos con patologías que precisen una restricción de las mismas como en insuficiencia renal, insuficiencia cardiaca o la hipertensión arterial.

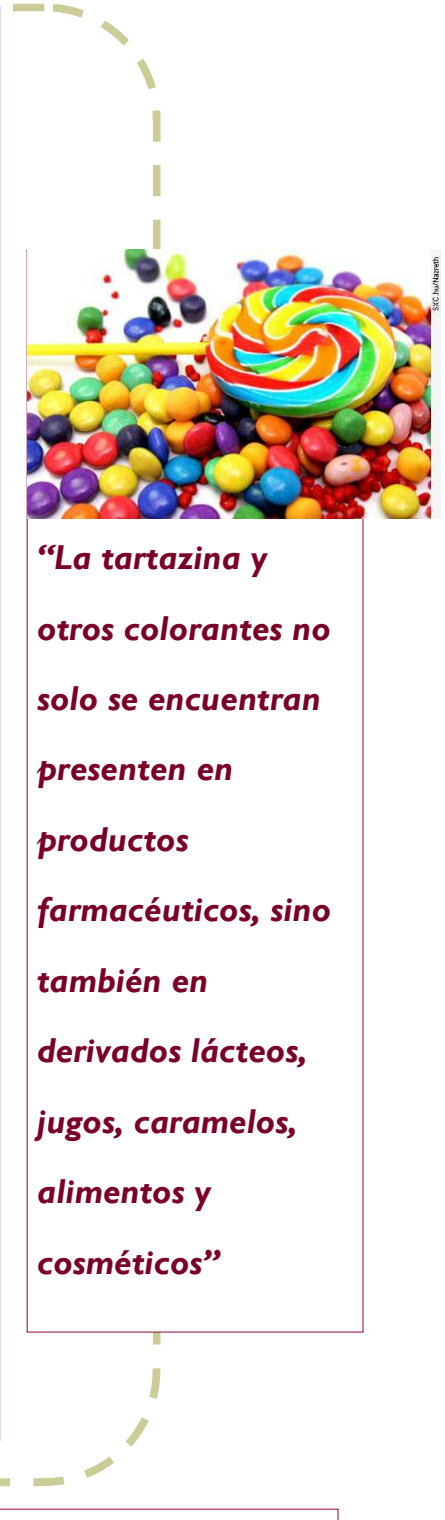

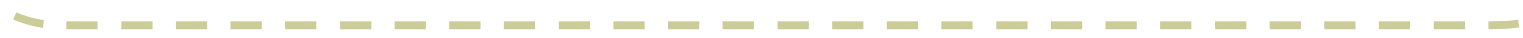

\section{Ejemplos y lista de excipientes de reporte obligatorio según la Agencia Europea del Medicamento}

\section{Caso \# I}

Se identifica que hay pacientes que prefieren un producto sobre otro que tienen idénticos principios activos. A menudo la preferencia se debe a que el paciente ha padecido una reacción adversa a uno de los medicamentos. Este fenómeno pasa desapercibido hasta que en 197I se registró un caso de cefaleas intensas y de alteraciones gastrointestinales prolongadas asociadas a la ingesta de Premarín $®$ (Estrógenos conjugados) en los Estados Unidos. En aquel momento la formulación de Premarín $®$ contenía 28 ingredientes, de los que solo el estrógeno conjugado se consideraba activo. Entre los 28 ingredientes se incluía un colorante amarillo comúnmente conocido como tartrazina.

Un médico (Dr. Lockey) confirmó que la paciente estaba reaccionando sólo a la tartrazina y pudo mediante el lavado del

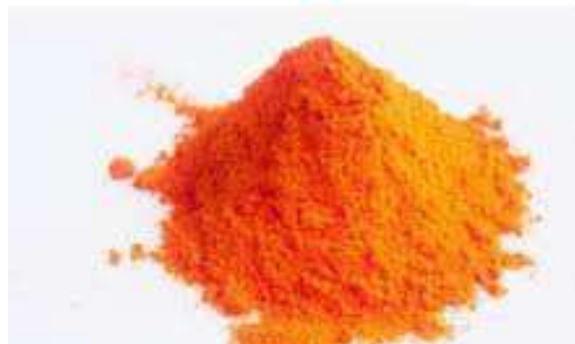

Tartazina: colorante amarillo FD\&C (Food Drug and CosmeticUSA) No 5 


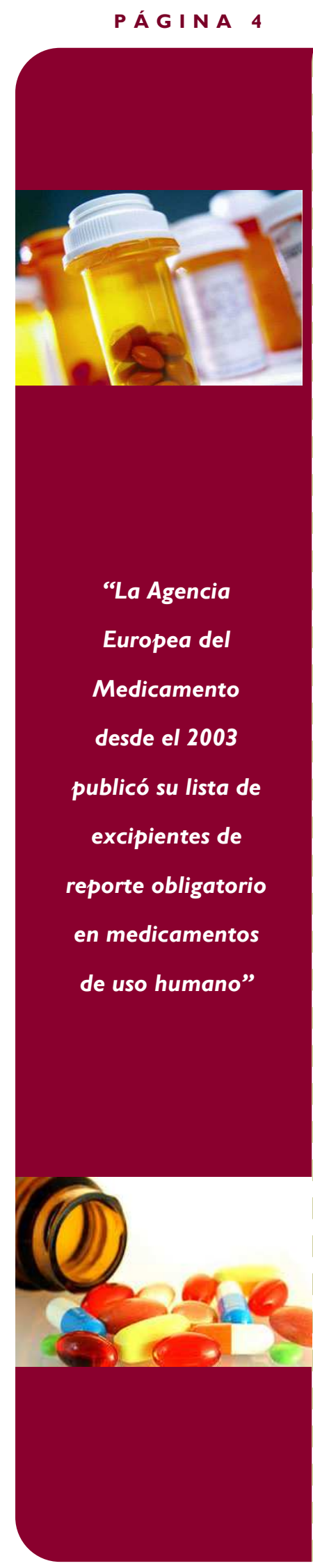

Ejemplos y lista de excipientes de reporte obligatorio según la Agencia

Europea del Medicamento

colorante de cada comprimido, seguir la administrando la medicación sin que se registraran más efectos adversos. Si Lockey no hubiera probado la reacción de la paciente a cada uno de los componentes de la formulación, casi seguro que los efectos adversos habrían sido atribuidos al principio activo.

\section{Caso \#2}

\section{"Tintura de Naranja"}

Paciente pediátrico con infecciones recurrentes de las vías respiratorias altas, hipersensible a penicilina y sus derivados.

Se prescribe eritromicina suspensión oral de la marca Erythrocin $125 \AA$ presentación de $25 \mathrm{mg} / \mathrm{mL}$

Inmediatamente después de la ingesta de la primera dosis el paciente presentó intenso dolor abdominal, náuseas y vómitos. Debido a que no se consideró la posibilidad de alergia a la eritromicina se sustituyó la medicación por Erythocin $250 \AA$ con presentación de $50 \mathrm{mg} / \mathrm{mL}$ y al ingerir esta presentación no se produjo ninguno de los síntomas previos.

Varios meses después se le vuelve a administrar al niño Erythrocin $125 \AA$ y volvió a presentar los mismo síntomas.

Una revisión de las historias clínicas del mismo centro hospitalario donde fue atendido el niño confirmaron que en los últimos 15 años , 16 pacientes habían manifestado el mismo tipo de intolerancia al Erythrocin $125 \AA$.

El laboratorio fabricante
(Abbott) reveló que la única diferencia entre las dos formulaciones de eritromicina era el colorante y aromatizante que es tintura de naranja (Erythrocin $125 \AA$ ) y jarabe de cerezas (Erythrocin 250®).

\section{Legislación}

En Europa hay un marco normativo desde 1982 en el que se indicaba que las especialidades farmacéuticas de uso humano en que figuren como excipientes tartrazina, etanol, lactosa, sulfitos, ácido bórico o boratos deberán indicar en el material de acondicionamiento y en el apartado "Composición" la presencia del excipiente en cuestión.

En el 2003 la Agencia Europea del Medicamento publica su guía "Excipientes in the label and Packaged leafelt of medicinal products for human use". (Etiquetado de excipientes en medicamentos de uso humano).

La siguiente es una lista de los excipientes de reporte obligatorio: Aprotinina

Aceite de maní

Aspartame

Agentes colorantes “Azo": tartrazina, azorubine, amaranto, la eritrosina, el rojo Ponceau, el amarillo anaranjado $S$

Bálsamo de Perú

Cloruro de Benzalconio

Ácido bórico y benzoatos: ácido benzoico, benzoato de sodio, benzoato de potasio

Alcohol bencílico

Aceite de bergamota

Bronopol

Butilato hidroxianisole

Butilato hidroxitolueno
Aceite de castor

Alcohol cetoestéarico

Clorocresol

Dimetil sulfóxico

Etanol

Formaldehído

Frutosa

Galactosa

Glucosa

Glicerol

Heparina (como excipiente)

Glucosa hidrogenada (jarabe)

Lactitol

Lactosa

Lanolina

Latex

Manitol

Compuestos orgánicos derivados del mercurio: timerosal, nitrato de fenilmercurio

Parahidroxibenzoatos $y$ sus ésteres

Fenilalanina

Potasio

Propilenglicol y sus ésteres

Aceite de sésamo

Sodio

Ácido sórbico y sus sales

Sorbitol

Aceite de soya

Alcohol esteárico

Sucrosa

Sulfitos y metasulfitos

Trigo

Lanolina

Xilitol 


\section{Ejemplos y lista de excipientes de reporte obligatorio según la Agencia Europea del Medicamento}

Es importante considerar que a pesar de que los excipientes indicados en la lista anterior implican su aparición en el etiquetado del medicamento, no necesariamente es así. Debido a que algunos de ellos están condicionados a la cantidad de excipiente en la formulación, tal es el caso de la sacarosa cuando la cantidad en una dosis sea máxima a 5 gramos se debe declarar.

En caso de excipientes que no sean de declaración obligatoria pero que exista evidencia de ser causa de reacciones adversas es recomendable que los fabricantes los empiecen a incluir en la sección de advertencias de los prospectos.

Las agencias reguladoras coinciden en que esta legislación proporcionará más protección a los pacientes.

En conclusión, desde un punto de vista físico una especialidad farmacéutica es la suma de cada uno de sus componentes activos o no, pero desde un punto de vista farmacológico un medicamento no incluye sus excipientes. sin embargo, está demostrado que no solo las sustancias activas son capaces de producir efectos adversos.

Al realizar la lectura de los ejemplos anteriores queda claro que la idea tradicional de inercia asociada a los excipientes no se ajusta a las condiciones reales.

También por lo general las reacciones adversas asociadas a excipientes son poco frecuentes $y$ poco graves en relación con las reacciones adversas producidas por los principios activos, a veces, es necesario que transcurran años de utilización para que se pueda demostrar la causalidad.
El farmacéutico tiene como función conocer y asesorar tanto al médico como al paciente respecto a los principios activos y excipientes presentes en las formulaciones $y$ las posibles reacciones adversas relacionadas con ellos.

Algunas veces se deja de utilizar un principio activo por no comprobar si la reacción adversas se debe en realidad a los excipientes.

Se debe recordar, que la máxima información disponible sobre excipientes se indica en el etiquetado $y$ prospecto del medicamento.

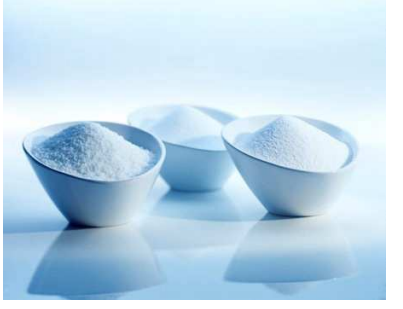

"La idea tradicional de inercia asociada a los excipientes no se ajusta a las condiciones reales"

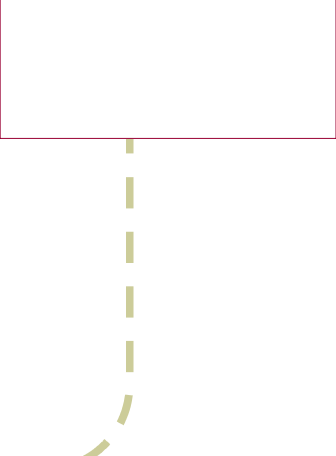

\section{Alertas de Farmacovigilancia Enero - Febrero 2013}

A continuación un resumen de las alertas de Farmacovigilancia más importantes emitidas por las agencias reguladoras internacionales durante los meses de enero y febrero del 2013.

Domperidona: la agencia australiana del medicamento a raíz de un estudio epidemiológico reciente recomienda que se inicie el tratamiento con domperdiona a las dosis más bajas posibles debido a que se encontró un aumento del riesgo de las arritmias ventriculares o casos de muerte súbita cuando el medicamento se usa a dosis superiores de $30 \mathrm{mg}$ en pacientes mayores de 60 años. Se recomienda a los pacientes interrumpir la medicación en caso de mareos, palpitaciones o convulsiones. También se recuerda a los profesionales que la domperidona está contraindicada con el uso concomitante de inhibidores del CYP3A4 como el ketoconazol, claritormicina, fluconazol, amiodarona, entre otros debido a que pueden prolongar el intervalo QT.

Fingolimod: la agencia australiana del medicamento emite una alertarecordatorio sobre los efectos cardiovasculares asociados a este medicamento y se contraindica su uso concomitante con antiarritmicos de Clase IA y Clase III. Además, al administrar Fingolimod por pri- mera vez, se requiere que el paciente sea monitorizado y realizar electrocardiograma.

Estatinas: la agencia canadiense informa sobre una actualización de la sección de seguridad del etiquetado de las estatinas, La actualización consiste en informar sobre el riesgo de aumento de presión arterial y el aumento de los niveles en sangre asociadas al uso de estatinas en pacientes con factores de riesgo de desarrollar diabetes. La agencia afirma que a pesar de esto, son mayores los beneficios observados que los efectos adversos reportados. 


\section{Alertas de Farmacovigilancia Enero - Febrero 2013}

Telaprevir: La FDA alerta sobre una serie de reportes de reacciones cutáneas severas producidas por Telaprevir, Este medicamento es utilizado en el tratamiento de la Hepatitis $C$ en combinación con peginterferon alfa y ribavirina. Esta alerta fue incluida en el etiquetado del producto y se informa que el tratamiento se debe suspender en caso de aparecer reacciones de este tipo.

Tolvaptan: la FDA informa sobre el potencial daño hepático asociado a este medicamento. En un estudio aleatorizado realizado en pacientes con enfermedad renal poliquística autosómica dominante, los pacientes expuestos al Tolvaptan desarrollaron un aumento significativo de la Alanino aminotransferasa (ALT). Estas alteraciones se observaron principalmente durante los primeros 18 meses de tratamiento. Al descontinuar el tratamiento los pacientes resolvieron positivamente. La FDA recomienda a los médicos tratantes realizar monitores periódicos de las enzimas hepáticas a pacientes con estos tratamientos $y$ especialmente en aquellos con fatiga, anorexia, ictericia y orina oscura. Si se sospecha daño hepático el medicamento debe ser suspendido.

Zolpidem: La FDA recomienda administrar dosis mínimas de este medicamento al acostarse debido a que se observado que algunos pacientes tiene niveles sanguíneos muy altos en la mañana siguiente, lo que les imposibilita realizar actividades que impliquen estar alerta. El riesgo es aún mayor en pacientes que toman la formulación de liberación retardada y también hay más riesgo en mujeres. La FDA espera más información y continua evaluando el riesgo de este medicamento y otros utilizados en insomnio y sus consecuencias en situaciones $\circ$ momentos del día que requieran mantenerse alerta.
Carbamazepina, oxcarbamazepina y eslicarbazepina: la agencia reguladora de medicamentos y productos sanitarios del Reino Unido informa sobre el potencial de desarrollar reacciones cutáneas serias asociadas a la presencia del alelo HLA-A* 310I y estos medicamentos. La presencia del alelo se ha reportado en pacientes europeos de origen o de descendencia japonesa. Los antígenos leucocitarios humanos están implicados en una respuesta inmunitaria anormal.

Entre las reacciones reportadas están: Síndrome de Steven Johnson, exantema pustuloso generalizado y el rash maculopapular. Aún no se ha determinado como medida regulatoria el hacer examen genético antes de recibir estos tratamientos, pero si se sugiere siempre establecer el riesgo- beneficio.

Ciproterona y progestágeno de tercera y cuarta generación: la Agencia Española del Medicamento ha iniciado una revisión de la relación riesgo- beneficio de medicamentos que contienen esta combinación. El uso de contraceptivos orales que contienen progestágenos de tercera o cuarta generación, como desogestrel, gestodeno o drospirenona, se asocia al doble de riesgo de enfermedad tromboembólica venosa (ETEV) en comparación con levonorgestrel

La agencia francesa del medicamento anunció la suspensión de la comercialización de preparados con esta combinación por esta misma causa desde enero de este año.

Antiepiléticos durante la gestación: El Instituto Catalán de Farmacología informa sobre un artículo publicado en The Lancet Neurology en enero de 2013 en el que se indica que la exposición fetal al ácido valproico se asocia a una reducción del cociente intelectual, dependiente de la dosis. en los niños a los 6 años de edad, según los resultados del estudio observacional prospectivo NEAD. Los resultados del análisis intermedio del estudio NEAD, publicados en 2009, mostraron que los niños de madres tratadas con ácido valproico durante la gestación presentaban una función cognitiva alterada a los 3 años de edad, en comparación con otros antiepilépticos. Un total de 224 niños completaron el seguimiento hasta los 6 años. Según los resultados definitivos del estudio, la exposición fetal al ácido valproico se asoció a un menor coeficiente intelectual a los 6 años (8 a II puntos menos) que con otros antiepilépticos, como la carbamacepina, la lamotrigina o la fenitoína. Esta afectación de la función cognitiva podría ser atribuible a cambios en las capacidades verbales inducidos por la exposición a los fármacos antiepilépticos.

Tetracepam: La Agencia Europea del Medicamento está revisando la información de seguridad de este medicamento, debido a un aumento de las notificaciones de reacciones cutáneas severas. La agencia establecerá próximamente la relación riesgo beneficio de este tratamiento debido a que la tasa de reacciones adversas es más elevada con este medicamento que con otras benzodiazepinas.

Denosumab: el Instituto Catalán de Farmacología alerta según un "Drug Safety Update de la Agencia reguladora de medicamentos y productos sanitarios del Reino Unido que existe un aumento del riesgo de fracturas femorales atípicas en pacientes con osteoporosis postmenopáusica en tratamiento prolongado con denosumab. Se notificaron dos casos confirmados de fractura atípica en pacientes tratados con denosumab $60 \mathrm{mg}$ durante 2,5 años o más. Se estima una incidencia de I a 10 por 10.000 pacientes tratados. Se recomienda informar a los pacientes y suspender el tratamiento en caso de sospecha de fractura atípica. 


\section{Bibliografía}

División de Farmacología Clínica. Instituto Catalán de Farmacología. Universidad Autónoma de Barcelona. SetiembreOctubre 1985. Butlletí Groc- Excipientes y aditivos: peligros ocultos de los medicamentos y en la sustitución de una marca por otra.

Guidelines Volume 3B. Medicinal products for human use. Safety, enviroment and information. Excipients in the label and package leaflet of medicinal productos for human use. European Comisión. Enterprise Directorate-General. Revision I. Brussels, ENTR/F2/BL D(2003). July 2003.

Viamote, A. 2007. Reacciones Adversas debidas a los excipientes. Centro de Información de Medicamentos. Colegio Oficial de Farmacéuticos de Navarra.

www.agemed.es/actividad/documentos/docs/circular20082.pdf

http://www.saludyfarmacos.org/boletin-farmacos/boletines/ jun2008/regulacion-y-politicas-europa/

Toda la bibliografía utilizada en este trabajo está a disposición de los lectores que la soliciten.

\section{Redacción y diseño}

\section{Dra. Cristina Fernández Barrantes}

\section{Farmacéutica}

\section{Hospital San Juan de Dios}

Centro de Información de Medicamentos

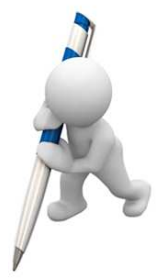

\section{Correspondencia}

farmacovigilanciahsjd@gmail.com cristifdez@gmail.com



Alertas de Farmacovigilancia

Agencia Europea del Medicamento:

http://www.ema.europa.eu/ema/index.jsp?curl=pages/

regulation/landing/regulation.jsp\&mid=

U.S. Food and Drug Adminsitration:

http://www.fda.gov/

Instituto Catalán de Farmacología: Butlletí Groc

http://w3.icf.uab.es/notibg/bgcat.php

Pharmaceutical Newsletter:

http://www.who.int/medicines/publications/newsletter/en/

Ediciones anteriores de este boletín en:

Página del colegio de Farmacéuticos

de Costa Rica

http://www.colfar.com

\section{Revista Clínica HSJD-Escuela de Medicina UCR}

http://revistaclinicahsjd.ucr.ac.cr
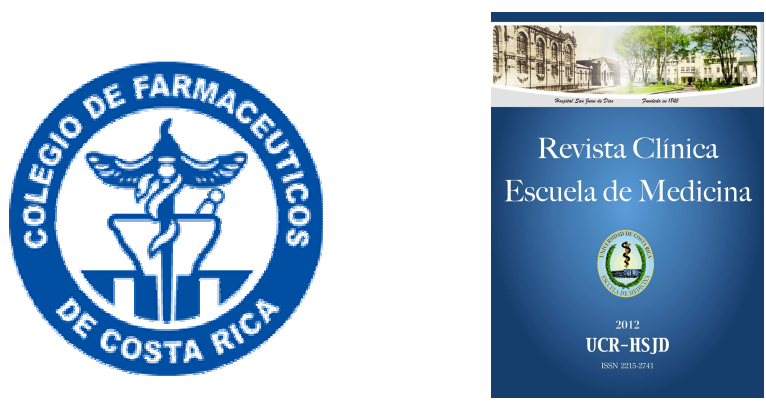